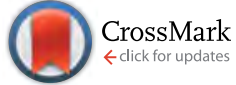

Cite this: RSC Adv., 2016, 6, 106090

Received 13th September 2016 Accepted 2nd November 2016

DOI: 10.1039/c6ra22872k

www.rsc.org/advances

\title{
Piezo-resistive and thermo-resistance effects of highly-aligned CNT based macrostructures
}

\begin{abstract}
V. T. Dau, $\dagger^{\text {*a }}$ C. D. Tran,,$^{\mathrm{b}}$ T. T. Bui, $\uparrow^{\mathrm{c}}$ V. D. X. Nguyen ${ }^{\mathrm{d}}$ and T. X. Dinh ${ }^{\mathrm{e}}$
Recent advances in assembling Carbon NanoTubes (CNTs) into macrostructures with outstanding properties, such as high tensile strength, high conductivity and porosity, and strong corrosive resistance, have underpinned potentially novel applications. For example, in advanced electronics, bioengineering and nanomechanics. This paper focuses on the development of (i) the piezoresistive polydimethylsiloxane-CNT (PDMS-CNT) composite membrane, and (ii) the thermo-resistive CNT hotwire using a technique of producing highly aligned CNT yarns and films. Our experimental results show that while PDMS-CNT films possess an outperformed gauge factor (10.7) compared with ones of CNT films in recent publications and several metals, a clear linear relationship of the resistance versus the temperature for a hotwire using CNT yarn is observed. Hence, the work supplies valuable evidence in the use of CNT films and yarns in several potential applications as thermal sensing elements and anemometric hotwires, respectively.
\end{abstract}

\section{Introduction}

Besides the characteristics of either a metallic conductor or semiconductor which allow carbon nanotubes (CNTs) to be used as a promising substitution for various components in integrated circuits to continue shrinking the transistor size, CNTs possess unique mechanical properties, for example, its tensile strength is much higher than any other known materials' ones. ${ }^{1}$ Thus, CNTs have been used in various applications including tunable electrometrical oscillators, ${ }^{2}$ chemical and physical devices, ${ }^{3}$ non-volatile memory, ${ }^{4}$ and a nanotube radio. ${ }^{5}$ In order to accurately deposit CNTs at a desired location, several methods have been investigated. Those techniques include the synthesized SWNTs which are preferentially attracted to the $\left(-\mathrm{NH}_{2}\right)$ functionalized surface, ${ }^{6,7}$ fluidic manipulation, ${ }^{8,9}$ dielectrophoretic manipulation in which CNTs are trapped and aligned by either alternative or discontinuous current fields $\mathbf{s}^{\mathbf{1 0 - 1 2}}$ and nano-robotic manipulation. ${ }^{13-18}$

An alternative approach is to assemble a massive quantity of CNTs into macrostructures using highly oriented CNTs to form

\footnotetext{
${ }^{a}$ Research Group (Environmental Health), Sumitomo Chemical. Ltd, Hyogo 665-8555, Japan

${ }^{b}$ School of Mechanical and Electrical Engineering, University of Southern Queensland, Queensland QLD 4350, Australia. E-mail: dauthanhvan@gmail.com; dauv@sc. sumitomo-chem.co.jp

${ }^{c}$ University of Engineering and Technology, Vietnam National University, Hanoi, Vietnam

${ }^{d}$ HCMC Institute for Computational Science and Technology, HCMC, Vietnam

${ }^{e}$ Graduate School of Science and Engineering, Ritsumeikan University, Kyoto, 5258577, Japan

$\dagger$ These authors contributed equally.
}

well-defined configurations. ${ }^{19}$ The highly oriented CNTs can be synthesized by the water-assisted chemical vapor deposition (CVD) technique. ${ }^{20}$ By the method, very long and catalyst-free SWNTs with diameter of $2.8 \mathrm{~nm}$ and high purity of carbon (greater than 99.9\%) are vertically aligned in form of sparse forest films. Owing to the large size, a CNT membrane can be used to cover on electrodes immersed in isopropyl alcohol (IPA) solution. A high-density SWNT membrane is then formed on electrodes' face after the IPA's volatilization by liquid-induced zippering effect. After positioning a CNT film at a desired position, the process can be integrated into developed technologies to create stretchable mechanical sensors, ${ }^{21,22}$ electronics devices. ${ }^{23}$ This technique also allows to produce large scale soft elastomeric electronics coated by a CNT film using the solution of ionic liquid mixed with fluorinated rubber in which CNTs are dispersed. ${ }^{24,25}$

Besides, highly aligned CNTs can be assembled in very large scales from spinnable CNT forest by pulling them to form longitudinal aligned film, ${ }^{26,27}$ or by pulling-twisting into CNT yarn. ${ }^{28,29}$ The CNT film possesses physical properties as presented in ref. 30. Such processes have been applied to fabricate the CNT based thread detection sensor for damage and the strain sensor of composite material. ${ }^{31-33}$ By the same method, CNT yarns and films were applied to electrical antenna, gas sensor. ${ }^{34,35}$ Another way to produce highly aligned yarns is to use the wet spinning method based on the chemical inertness and agglomeration of CNTs dispersed in super-acids. ${ }^{36,37} \mathrm{CNT}$ filaments are then produced by extruding super-acids through a spinneret into a coagulation solution such as water or acetone. ${ }^{38,39}$ The CNT filaments by this method have good electrical and thermal conductivities and were investigated their application as capacitive electrodes. ${ }^{\mathbf{4 0}}$ 
While CNT has been synthesized and then commercialized in bundle form, the development of applications of CNT based macro scale is always an interesting challenge. Since the technique of assembling CNTs into a macro scale ultimately affects the characteristics of CNT macrostructures, the applications were investigated and reported accordingly for each method. Meanwhile the CNT membrane is promising in applications requiring large deformation due to its flexibility, the CNT yarn is more suitable in various electronic applications owing to its high intensity and good thermal and electrical conductivities.

In this paper, the technique of producing highly aligned CNT yarns and films by Tran and his co-workers ${ }^{\mathbf{4 1 , 4 2}}$ will be exploited to develop (i) the piezoresistive polydimethylsiloxane-CNT (PDMS-CNT) composite membrane for mechanical sensing application, and (ii) the thermo-resistive hotwire made of spinnable CNT yarn. Together with the publications related to the piezoresistive effect using CNT thread by this approach ${ }^{43}$ and other physical properties of CNT membrane, ${ }^{30}$ our present work contributes to understanding the piezoresistive effect in form of PDMS-CNT membrane and demonstrate an instant application of CNT yarn as a promising hotwire element. Thus, thanks to the high conductivity and porosity and the strong corrosive resistance, these CNT based structures can be useful in several specific applications. Some applications can be cited as follows: the electro-hydrodynamics where CNT threads act as a positive spraying electrode to overcome the corrosion induced by the electrochemical process, ${ }^{\mathbf{4 4 , 4 5}}$ the corona electrode for ion wind generator, ${ }^{46-51}$ the thermal sensing element in multiphase fluidic device $^{52}$ and the large deformation sensor and actuator. ${ }^{22,53}$

\section{CNT fabrication process}

Among techniques for the synthesis of CNTs, the CVD method is widely used. ${ }^{54-56}$ By this technique, the parameters of CNTs growing up on a wafer can be controlled by changing the thickness of the catalyst layer, the processing time in the reactor and other additional processing. ${ }^{26} \mathrm{~A}$ floating catalyst CVD method has been successfully applied to the production of spinnable CNT forests using a two-stage synthesis process. ${ }^{57}$ For this scheme, CNT arrays were grown on silica using ferrocene as catalyst precursor and cyclohexane as solvent and carbon source..$^{57,58}$ The main advantages of the floating catalyst method include (i) not to require any complex equipment and (ii) to obviate the need for a preparation of catalyst layer on the substrate.

In this research, the spinnable CNT forest is pulled out into a film/web (Fig. 1a) which is then twisted into yarns (Fig. 1b and c). ${ }^{41}$ As presented in Section 1, CNT film and yarn are the objects for the characterization process in this paper.

\section{Experimental setup}

\section{Piezoresistive effect of CNT film}

PDMS is a polymer widely used in bio-medical applications including the components used in microfluidics, implants, orthopedics and micro gaskets. For the experiment, the PDMS solution mixed with a curing agent with 10/1 ratio is firstly applied on the prepared silicon wafer and degassed until the

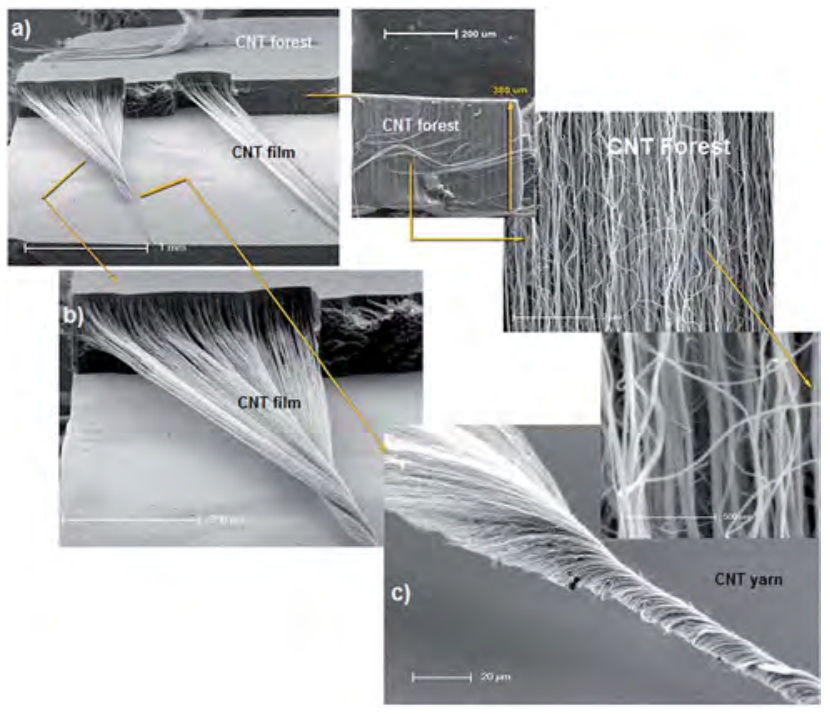

Fig. 1 CNT film and yarn: manufacturing process.

foam on surface disappears. After two copper electrodes are located at desired positions on the PDMS membrane, an aligned CNT membrane pulled out from the CNT forest is then laid on the PDMS film. A thin layer of silver pasted conductive epoxy is coated on the location of electrodes to ensure their electric contact. This process is carried out using the three multi-axis movable jigs Opto-Sigma with the resolution of $0.05 \mathrm{~mm}$, one for CNT forest, one for pulling CNT film, and the last one for electrodes. Finally, PDMS is casted one more time to cover the sample. Since the thickness of electrodes causes a gap between the CNT web and initial PDMS film, PDMS moves underneath to form a PDMS film embedding the CNT membrane in the middle layer as presented in Fig. 2a. The CNT-PDMS film is heated at $70{ }^{\circ} \mathrm{C}$ during 6 hours and then peeled out from the wafer at the room temperature (Fig. 2b). Fig. 3a and b depict a fabricated PDMS-CNT film using the presented process.

The PDMS embedded CNT membrane is installed longitudinally using the IMADA force measurement system. In order to measure the resistance of the CNT membrane, the external leading wires are connected to a silver paste interconnection which facilitates the four-point resistance measurement to eliminate unexpected contact resistance. The PDMS specimen is hold by two clamps, one is fixed and the other one is movable which is pulled with a constant velocity (see Fig. 3c). The length variable of the specimen between the fixed and movable clamps and its corresponding resistance are recorded and the relationship between the resistance and strain of the PDMS-CNT film is established. The measurements were carried out at $24{ }^{\circ} \mathrm{C}$ and less than $55 \%$ relative humidity in laboratory to minimize the influence of humidity on the inter-SWCNT junction which affects significantly the CNT network resistance..$^{59,60}$

\section{Thermoresistive effect of CNT yarn}

CNT membrane pulled out from the forest is twisted into a yarn whose thermo-resistive effect will be characterized to illustrate 

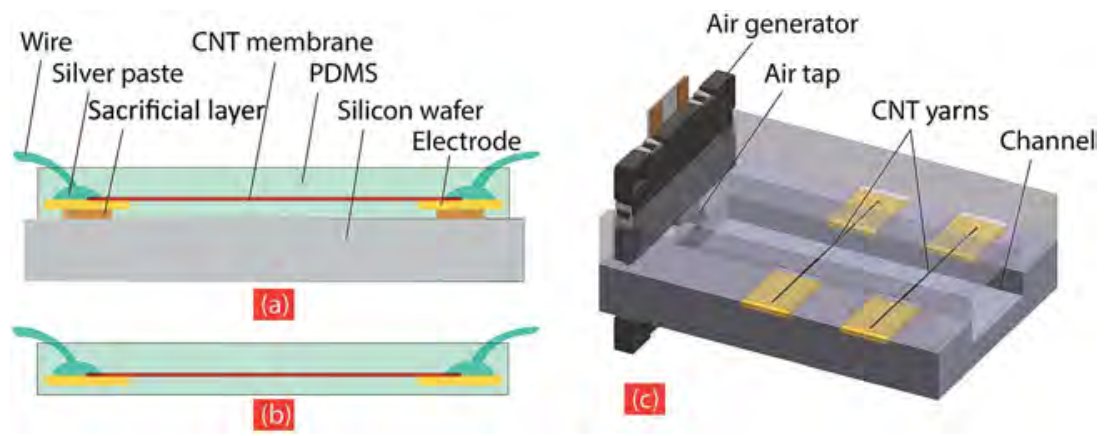

Fig. 2 Piezoresistive effect of CNT film: schema of CNT embedded PDMS film ( $a$ and b), and thermo-resistive effect of CNT yarn: schema of fluidic device and experiment set-up (c).
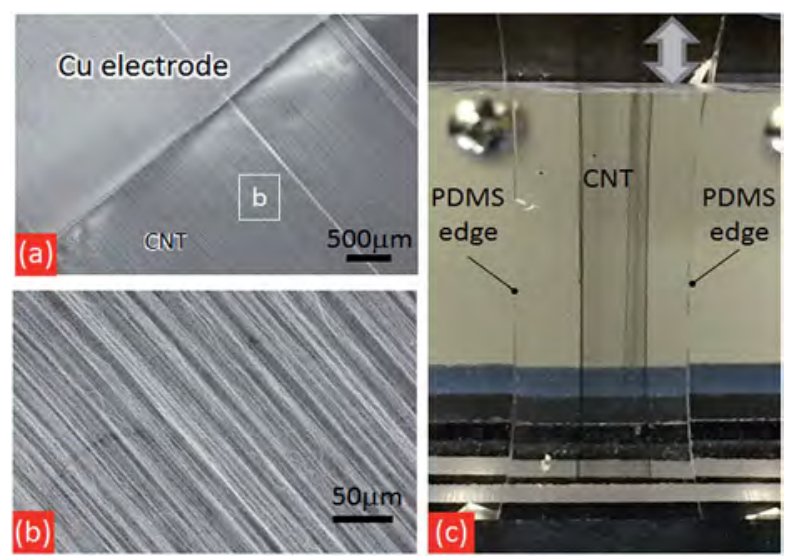

Fig. 3 Piezoresistive effect of CNT film: SEM images of PDMS-CNT film ( $a \& b$ ) and PDMS-CNT firm under tensile loading (c).

the potential application of CNT yarn as a thermal sensing element. In order to determine this feature of CNT yarn, a simple fluidic device including air generator as presented in ref. 61 and a millimeter scale channel are prepared. The experiment is set up as follows. With the use of movable jigs, two stretched yarns are placed across a channel between two metal tags as described in Fig. 2c. The electrical connection is similarly processed as measuring piezoresistive effect of CNT film. The experiment is carried out in a temperature controlled chamber to measure the temperature coefficient of temperature (TCR) of CNT yarn. In the test of hotwire anemometry device, the air flow is supplied by an air generator whose tap is placed $22 \mathrm{~mm}$ from the yarn. The CNT yarn is heated by a constant current and the measured outputs include the voltage and current. The air flow is calibrated by a conventional flow meter of Shibata.

\section{Experimental results of piezo- resistive effect for CNT film and discussions}

A prepared PDMS-CNT film under tensile test is shown in Fig. 3c. It can be seen that CNT film was covered completely by PDMS using the experimental set-up as described in Section 3.
With the initially unstretched length of $30 \mathrm{~mm}$, the resistance of the CNT film is measure to be $110 \mathrm{k} \Omega$.

The piezo-resistive sensing property of a specimen represents the change of its resistance with respect to the specimen's deformation. The piezoresistive sensing property is characterized by the gauge factor (GF) which is defined as the change in resistance $\mathrm{d} R / R_{0}$ of a specimen divided by its strain $\varepsilon, \mathrm{GF}=\left(\mathrm{d} R / R_{0}\right) / \varepsilon$.

Fig. 4 presents the change of resistance of CNT film with respect to its elongation. The experimental results show that the resistance has a high repeatability with the CNT film's deformation. The changes of resistance for three repeats are the same with the strain $\varepsilon$ increasing to $4 \%$. Although the test should be done longer with more repeated cycles, the repeatability for full scale from $0 \%$ to $4 \%$ deformation has achieved good repeatability. The test was then further extended for damaging limit, which is found to be around $5 \%$.

Recently, although a PDMS-CNT films by Yamada's group ${ }^{22}$ advised a strain of $280 \%$, this result is based on a CNT macrostructure which was processed to form a waving shape in the film. This waving structure gears down the CNT component's train against the PDMS-CNT film one. Indeed, by the structure, the PDMS-CNT films can be pulled largely while CNT macro-structure

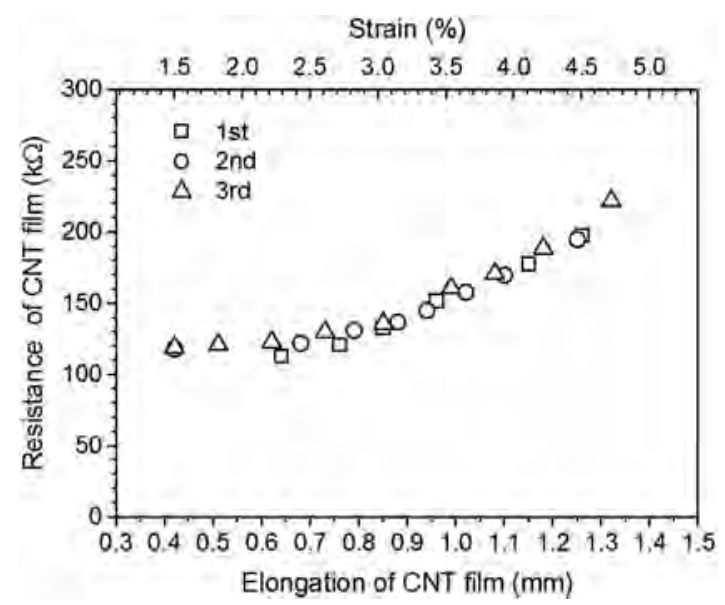

Fig. 4 Piezoresistive effect of CNT film: relationship between the resistance and elongation of CNT film under tensile load. The experiment was repeated with 3 times using initially unstretched length of CNT films of $30 \mathrm{~mm}$. 

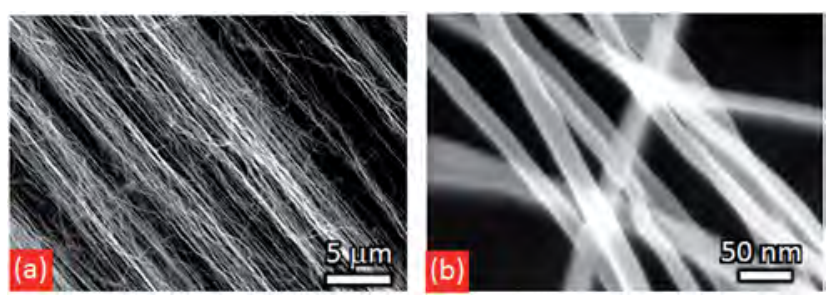

Fig. 5 Piezoresistive effect of CNT film: SEM images of a fabricated PDMS-CNT film.

is stretched much less. Thus, this trade-off reduces the GF of PDMS-CNT film one order of magnitude, ranged from 0.06 to 0.82 .

The average GF of our CNT film is around 10.7 and hence outperformed compared with ones of other highly aligned CNT films, such as the result using the water-assisted CNT film whose GFs cover a range from 0.27 to $6.24 .^{62}$ This increase of GF can be explained as the improvement of CNTs' alignment in film due to a relevant initial stretch using the process presented in Section 3. Although the GF of our present CNT films is also larger than those of metals, for example copper with the range of GF from 2 to 5 , it is smaller than that of silicon-based sensors with a GF of 150-200 (ref. 63) and much lower than one of individual CNT-based piezoresistive strain sensors whose GF has overpassed 1000 as published in several applications. ${ }^{64,65}$

In strain sensing application, the piezoresistive effect of a CNT film is primarily dependent on the CNT's intrinsic resistance and inter-single CNT resistance. ${ }^{66}$ The inter-single CNT resistance consists of the resistance between tubes and the resistance caused by very small gap between tubes. The piezoresistive effects of a carbon nanotube film are primarily due to the modification of those resistances. Since CNT fibers are not perfectly aligned, when the film is stretched the gaps between CNTs may narrow and yields an increase of the physical contact between CNTs which reduce the resistance of the film. In addition, the resistance by CNT fibers whose directions are not on the load one (Fig. 5a and b) does not change significantly with the stretch of film. Furthermore, the CNT film pulled out of the wafer in our sample is not fully orientated. When stretching the CNT membrane, the entangled fibers are just aligned but not elongated to provide the piezoresistive effect. Thus, while the resistance of individual CNTs increases with the stretch, the random configuration of CNTs network in film would influence on the resistance of inter-single CNTs and then yields a lower GF of film.

\section{Results of thermo-resistive effect for CNT yarn and discussion}

Fig. 6b describes the preparation of two stretched CNT yarns which are set up to connect to an electrical circuit. A blower is assembled to the fluidic device as presented in Fig. 2c. The SEM images of CNT yarns and its covering conductive paste electrode are showed in Fig. $6 \mathrm{c}$ and d. It is noted that a similar process but without using conductive paste by Koechlin et al. ${ }^{67}$ stated a very small specific contact resistance in order of $\mu \Omega \times \mathrm{cm}^{2}$.
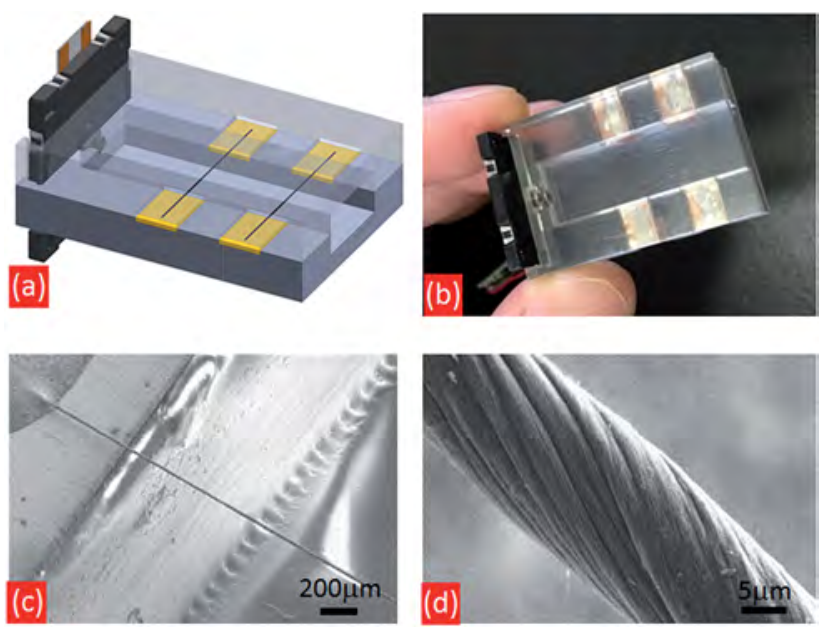

Fig. 6 Thermo-resistive effect of CNT yarn: half-view of test device showing CNTs yarns are placed across the channel, width of $10 \mathrm{~mm}$ (a $\&$ b). Zoomed CNT connecting electrode and conductive paste covering CNT yarns (c) and SEM image of CNT yarn as a hotwire sensing element $(d)$.

The ohmic contact was confirmed by a linear relation of the current and voltage as shown in Fig. 7-inset which is measured by a semiconductor parameter analyzer using the Agilent HP4155B. The resistances of the two yarns at $25{ }^{\circ} \mathrm{C}$ are approximately $2.5 \mathrm{k} \Omega$, slightly different because the effective lengths are varied due to electrical connection from the conductive paste. The change of the resistance with respect to the temperature for the two CNT yarns is presented in Fig. 7 where a linear relationship is observed for both CNT yarns. The two lines are parallel each other, with a narrow gap due to a little difference of their initial resistance, give similar temperature coefficient of temperature (TCR) of $-1200 \mathrm{ppm}^{\circ} \mathrm{K}^{-1}$.

In order to demonstrate the anemometry characteristic of CNT yarn, an air blower generator driven by a range of voltage from $5 \mathrm{~V}$ to $14 \mathrm{~V}$, corresponding to the flow rate of $0.3 \mathrm{l} \mathrm{min}^{-1}$ to $0.91 \mathrm{~min}^{-1}$ was installed with two CNT yarns acting as hotwire

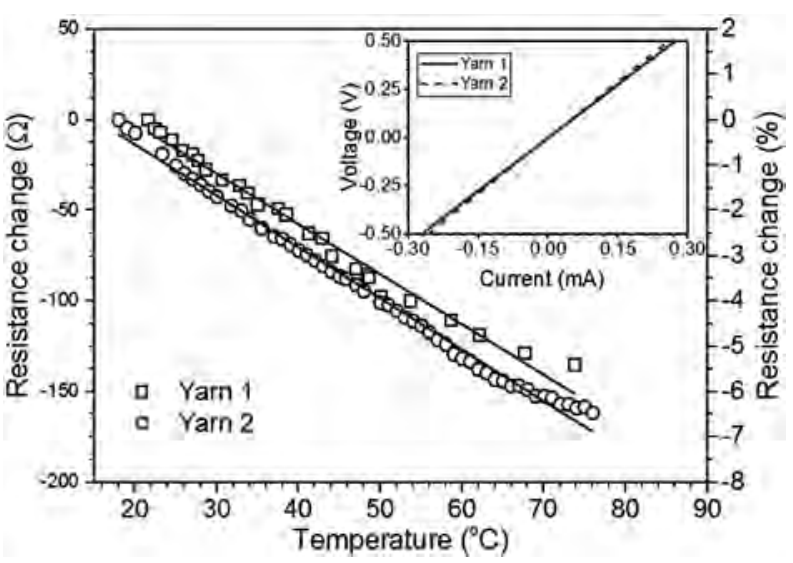

Fig. 7 Thermo-resistive effect of CNT yarn: relationship of the resistance of two yarns with respect to temperature. The inset shows the current-voltage characteristics of the yarn. 


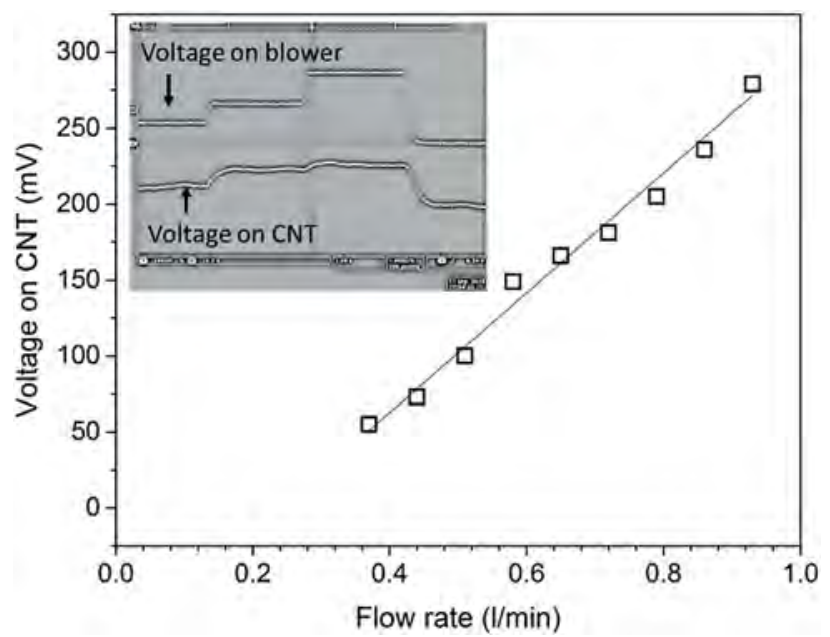

Fig. 8 Thermo-resistive effect of CNT yarn: the voltage measured between two ends of the CNT yarn hotwire is plotted versus the flow rate. The inset presents the time resolved data of output voltage on the CNT yarn versus the driving voltage on the blower.

as described in Section 3. The yarns are heated by constant current of $4.2 \mathrm{~mA}$ at room temperature.

Owing to the cooling by air from the blower, the CNT yarns reach the thermal equilibrium state at a desired temperature. The thermal equilibrium yields an increase of the resistance of CNT yarns and then a corresponding increase of the voltage. ${ }^{68-70}$ The output voltage of CNT yarns versus the flow rate generated by the air blower shown in Fig. 8 confirms a clear linearity of CNT yarn anemometry. The time resolved-data, recorded by the oscilloscope (Keysight 3012T) presented in the inset of Fig. 8 also shows a good response between the blower driving voltage and the output voltage on the hotwire. It is also worth noting that a development of flexible heating element by ref. 71 using unaligned CNTs mixed with PDMS also confirmed a qualitatively negative temperature coefficient behavior for TCR with nonlinearity of the heating process.

Our experimental results show that the conductivity of CNT yarn is one order of magnitude higher than that of CNT film with better repeatability for samples thanks to high density of CNT yarns. Indeed, by our experiment with the same length, the resistance of CNT film is around 10 times higher than one of CNT yarn (Fig. 4 and 7). Together with high conductivity, CNT yarn's properties of high tensile strength, high porosity and strong resistance to corrosive electrolyte have made possible novel applications, for example, the sensing element for inertial fluidic, electro-hydrodynamics and electrolytic applications.

\section{Conclusion}

The paper reports two promising properties of highly-oriented CNT based products, the piezoresistive effect of film for large strain applications and the thermo-resistive effect of yarn in the hotwire anemometry based on experimental results. Indeed, while PDMS-CNT films by the present work process an average GF (around 10.7) which is higher than ones of CNT films by recent publications and several metals, a clear linear relationship of the resistance versus the temperature for hotwire using CNT yarn is observed. The work supplies valuable evidences in the use of CNT films and yarns in several potential applications as thermal sensing elements and anemometric hotwires, respectively.

\section{References}

1 J.-P. Salvetat, G. Briggs, J.-M. Bonard, R. Bacsa, A. Kulik, T. Stöckli, N. Burnham and L. Forró, Phys. Rev. Lett., 1999, 82, 944-947.

2 V. Sazonova, Y. Yaish, H. Üstünel, D. Roundy, T. A. Arias and P. L. McEuen, Nature, 2004, 431, 284-287.

3 C. Xue, G.-Q. Du, L.-J. Chen, J.-G. Ren, J.-X. Sun, F.-W. Bai and S.-T. Yang, Sci. Rep., 2014, 4, 5925.

4 T. Rueckes, Science, 2000, 289, 94-97.

5 K. Jensen, J. Weldon, H. Garcia and A. Zettl, Nano Lett., 2007, 7, 3508-3511.

6 J. Liu, M. J. Casavant, M. Cox, D. A. Walters, P. Boul, W. Lu, A. J. Rimberg, K. A. Smith, D. T. Colbert and R. E. Smalley, Chem. Phys. Lett., 1999, 303, 125-129.

7 J. C. Lewenstein, T. P. Burgin, A. Ribayrol, L. A. Nagahara and R. K. Tsui, Nano Lett., 2002, 2, 443-446.

8 Y. Huang, Science, 2001, 291, 630-633.

9 W. Salalha and E. Zussman, Phys. Fluids, 2005, 17, 63301.

10 K. Yamamoto, S. Akita and Y. Nakayama, J. Phys. D: Appl. Phys., 1998, 31, L34-L36.

11 J. Chung, K.-H. Lee, J. Lee and R. S. Ruoff, Langmuir, 2004, 20, 3011-3017.

12 S. Evoy, N. DiLello, V. Deshpande, A. Narayanan, H. Liu, M. Riegelman, B. R. Martin, B. Hailer, J. C. Bradley, W. Weiss, T. S. Mayer, Y. Gogotsi, H. H. Bau, T. E. Mallouk and S. Raman, Microelectron. Eng., 2004, 75, 31-42.

13 T. Fukuda, F. Arai and L. Lixin Dong, Proc. IEEE, 2003, 9, 1803-1818.

14 J. Hone, in Carbon Nanotubes, Springer Berlin Heidelberg, Berlin, Heidelberg, 2001, pp. 273-286.

15 E. W. Wong, P. E. Sheehan and C. M. Lieber, Science, 1997, 277, 1971-1975.

16 H. W. C. Postma, A. Sellmeijer and C. Dekker, Adv. Mater., 2000, 12, 1299-1302.

17 M. R. Falvo, R. M. Taylor, a. Helser, V. Chi, F. P. Brooks, S. Washburn and R. Superfine, Nature, 1999, 397, 236-238.

18 M. Yu, Science, 2000, 287, 637-640.

19 Y. Hayamizu, T. Yamada, K. Mizuno, R. C. Davis, D. N. Futaba, M. Yumura and K. Hata, Nat. Nanotechnol., 2008, 3, 289-294.

20 K. Hata, D. N. Futaba, K. Mizuno, T. Namai, M. Yumura and S. Iijima, Science, 2004, 306, 1362-1364.

21 V. T. Dau, D. V. Dao, T. Yamada, B. T. Tung, K. Hata and S. Sugiyama, Smart Mater. Struct., 2010, 19, 75003.

22 T. Yamada, Y. Hayamizu, Y. Yamamoto, Y. Yomogida, A. Izadi-Najafabadi, D. N. Futaba and K. Hata, Nat. Nanotechnol., 2011, 6, 296-301.

23 C. Subramaniam, T. Yamada, K. Kobashi, A. Sekiguchi, D. N. Futaba, M. Yumura and K. Hata, Nat. Commun., 2013, 4, 2202. 
24 A. Sekiguchi, F. Tanaka, T. Saito, Y. Kuwahara, S. Sakurai, D. N. Futaba, T. Yamada and K. Hata, Nano Lett., 2015, 15, 5716-5723.

25 T. Sekitani, Y. Noguchi, K. Hata, T. Fukushima, T. Aida and T. Someya, Science, 2008, 321, 1468-1472.

26 K. Liu, Y. Sun, L. Chen, C. Feng and X. Feng, Nano Lett., 2008, 8, 700-705.

27 K. Liu, Y. Sun, R. Zhou, H. Zhu, J. Wang, L. Liu, S. Fan and K. Jiang, Nanotechnology, 2010, 21, 45708.

28 M. B. Jakubinek, M. B. Johnson, M. A. White, C. Jayasinghe, G. Li, W. Cho, M. J. Schulz and V. Shanov, Carbon, 2012, 50, 244-248.

29 M. B. Jakubinek, M. A. White, G. Li, C. Jayasinghe, W. Cho, M. J. Schulz and V. Shanov, Carbon, 2010, 48, 3947-3952.

30 J.-H. Pöhls, M. B. Johnson, M. A. White, R. Malik, B. Ruff, C. Jayasinghe, M. J. Schulz and V. Shanov, Carbon, 2012, 50, 4175-4183.

31 J. L. Abot, Y. Song, M. J. Schulz and V. N. Shanov, Compos. Sci. Technol., 2008, 68, 2755-2760.

32 J. L. Abot, Y. Song, M. S. Vatsavaya, S. Medikonda, Z. Kier, C. Jayasinghe, N. Rooy, V. N. Shanov and M. J. Schulz, Compos. Sci. Technol., 2010, 70, 1113-1119.

33 J. L. Abot, M. J. Schulz, Y. Song, S. Medikonda and N. Rooy, Smart Mater. Struct., 2010, 19, 85007.

34 S. D. Keller, A. I. Zaghloul, V. Shanov, M. J. Schulz and D. B. Mast, IEEE Antenn. Wireless Propag. Lett., 2013, 12, 1192-1195.

35 A. Hehr, Y. Song, B. Suberu, J. Sullivan, V. Shanov, M. Schulz and J. Youtcheff, in Nanotube Superfiber Materials, 2014, pp. 671-712.

36 A. B. Dalton, S. Collins, E. Muñoz, J. M. Razal, V. H. Ebron, J. P. Ferraris, J. N. Coleman, B. G. Kim and R. H. Baughman, Nature, 2003, 423, 703.

37 L. M. Ericson, H. Fan, H. Peng, V. A. Davis, W. Zhou, J. Sulpizio, Y. Wang, R. Booker, J. Vavro, C. Guthy, A. N. G. Parra-Vasquez, M. J. Kim, S. Ramesh, R. K. Saini, C. Kittrell, G. Lavin, H. Schmidt, W. W. Adams, W. E. Billups, M. Pasquali, W.-F. Hwang, R. H. Hauge, J. E. Fischer and R. E. Smalley, Science, 2004, 305, 1447-1450.

38 V. A. Davis, N. G. Parra-Vasquez, M. J. Green, P. K. Rai, N. Behabtu, V. Prieto, R. D. Booker, J. Schmidt, E. Kesselman, W. Zhou, H. Fan, W. W. Adams, R. H. Hauge, J. E. Fischer, Y. Cohen, Y. Talmon, R. E. Smalley and M. Pasquali, Nat. Nanotechnol., 2009, 4, 830-834.

39 X. Wang, N. Behabtu, C. C. Young, D. E. Tsentalovich, M. Pasquali and J. Kono, Adv. Funct. Mater., 2014, 24, 3241-3249.

40 F. Liu, R. M. Wagterveld, B. Gebben, M. J. Otto, P. M. Biesheuvel and H. V. M. Hamelers, Colloids and Interface Science Communications, 2014, 3, 9-12.

41 C. D. Tran, W. Humphries, S. M. Smith, C. Huynh and S. Lucas, Carbon, 2009, 47, 2662-2670.

42 C. D. Tran, in Nanotube Superfiner Materials, changing engineering design, ed. M. J. Schulz, V. N. Shanov and Z. Yin, Elsevier, 1st edn, 2014, pp. 211-242.
43 C. D. Tran, S. Lucas, D. G. Phillips, L. K. Randeniya, R. H. Baughman and T. Tran-Cong, Nanotechnology, 2011, 22, 145302.

44 R. P. Caramit, A. S. Antunes Araújo, D. K. Fogliatto, L. H. Viana, M. A. Gonçalves Trindade and V. S. Ferreira, Anal. Methods, 2015, 16-20.

45 T. Ohtsuka, Int. J. Corros., 2012, 2012, 1-7.

46 K. J. Lee, M. Jo, N. T. Hong, S. Lee, J. S. Oh, K. W. Jung and S. S. Yang, Jpn. J. Appl. Phys., 2014, 53, 36703.

47 V. T. Dau, T. X. Dinh, T. T. Bui and T. Terebessy, Flow Meas. Instrum., 2016, 50, 252-260.

48 V. T. Dau, T. X. Dinh, T. Terebessy and T. T. Bui, Sens. Actuators, A, 2016, 244, 146-155.

49 V. T. Dau, T. T. Bui, T. X. Dinh and T. Terebessy, Sens. Actuators, A, 2016, 237, 81-90.

50 V. T. Dau, T. X. Dinh, T. T. Bui, C. D. Tran, H. T. Phan and T. Terebessy, Exp. Therm. Fluid Sci., 2016, 79, 52-56.

51 V. T. Dau, T. X. Dinh, T. Terebessy and T. T. Bui, IEEE Trans. Plasma Sci., 2016, 1-9.

52 V. T. Dau, T. X. Dinh and T. T. Bui, Sens. Actuators, B, 2015, 223, 820-826.

53 V. T. Dau, B. T. Tung, T. X. Dinh, D. V. Dao, T. Yamada, K. Hata and S. Sugiyama, J. Micromech. Microeng., 2013, 23, 75024.

54 T. W. Ebbesen, Carbon nanotubes: preparation and properties, CRC Press, 1997.

55 M. S. Dresselhaus and P. Avouris, in Carbon Nanotubes, Springer Berlin Heidelberg, Berlin, Heidelberg, pp. 1-9.

56 M. S. Dresselhaus and M. Endo, in Carbon Nanotubes, Springer Berlin Heidelberg, Berlin, Heidelberg, pp. 11-28.

57 C. P. Huynh and S. C. Hawkins, Carbon, 2010, 48, 1105-1115.

58 S. C. Hawkins, J. M. Poole and C. P. Huynh, J. Phys. Chem. C, 2009, 113, 12976-12982.

59 Z. J. Han and K. Ostrikov, Appl. Phys. Lett., 2010, 96, 9-12.

60 K. Zhang, J. Zou and Q. Zhang, Nanotechnology, 2015, 26, 455501.

61 Murata Ltd.

62 V. T. Dau, T. Yamada, D. Viet Dao, B. Thanh Tung, K. Hata and S. Sugiyama, Microelectron. J., 2010, 41, 860-864.

63 M. J. Madou, Fundam. Microfabr. Sci. Miniaturization, 2002, p. 49.

64 C. Stampfer, A. Jungen, R. Linderman, D. Obergfell, S. Roth and C. Hierold, Nano Lett., 2006, 6, 1449-1453.

65 J. Cao, Q. Wang and H. Dai, Phys. Rev. Lett., 2003, 90, 157601. 66 P. L. Fuhrer, M. S. Nygard, J. Shih, L. Forero, M. Yoon, Y. G. Mazzoni, M. S. C. Choi, H. J. Ihm, J. Louie, S. G. Zettl and A. McEuen, Science, 2000, 288, 494-497.

67 C. Koechlin, S. Maine, S. Rennesson, R. Haidar, B. Trétout, A. Loiseau and J.-L. Pelouard, C. R. Phys., 2010, 11, 405-410.

68 D. V. Dao, V. T. Dau, T. Shiozawa, H. Kumaga and S. Sugiyama, in 19th IEEE International Conference on Micro Electro Mechanical Systems, IEEE, 2006, pp. 594-597.

69 I. Mabuchi and T. Tanaka, Bull. JSME, 1967, 10, 808-816.

70 V. T. Dau, D. V. Dao, T. Shiozawa and S. Sugiyama, IEEE Sens. J., 2008, 8, 1530-1538.

71 K. Chu, D. Kim, Y. Sohn, S. Lee, C. Moon and S. Park, IEEE Electron Device Lett., 2013, 34, 668-670. 